\title{
Study on structure stability based on image quality guarantee for GEO high resolution optical imaging satellite
}

\author{
ZHAO Hua ${ }^{*}, Z H A O$ YunPeng ${ }^{*}, W A N G$ ChengLun, WANG ZheYu and YANG Hua \\ Beijing Institute of Spacecraft System Engineering, Beijing 100094, China
}

\begin{abstract}
By contrast to one on low earth orbit (LEO), high spatial resolution optical imaging earth observation satellite on geostationary orbit has some remarkable advantages with much higher time resolution,more wider imaging field much longer target tracking and monitoring time,and now is becoming one of important development foucs. Corresspondently GEO optical imaging satellite has much higher control accuracy requirement with stability than LEO,greatly promote development difficulties.The paper makes dicussion for key structure influence factors on attitude \& structure stability for image quality guarantee under complex space circumstance and conducts stimulation to account for the their effect,finally present some solution options.
\end{abstract}

\section{Introduction}

GEO earth observation optical imaging satellite could levitate above the observation area and its centroid keep still relative to earth, it has a remarkable advantage of high time resolution over one on low earth orbit (LEO), and also higher efficiency of using. As progress of technology of large spatial camera development, spatial resolution is increasing quickly which provide a good technical basis for practical engineering application. Currently,GEO high resolution optical imaging satellite is becoming one of the focus of research \& development. China launched successfully a GEO optical imaging satellite with $50 \mathrm{~m}$-spatial resolution in 2015 , and has collected a number of high quality pictures which are being applied in fields including nature disaster and environment pollution monitoring, land resource survey, weather forcast and climate research, etc[1]. Last decade, Europe has been developing a series of projects about GEO optical imaging satellite such as GEO-Afiraca with 25m-spatial resolution, Geo-Oculus with $10 \mathrm{~m}$-spatial resolution, HRGeo concept deisgning with over3m-spatial resolution (ASTRUM). American is implementing JWST project with $\phi 6.5 \mathrm{~m}$ of primary mirror diamate, and developing a higher than $2.5 \mathrm{~m}$ spatial resolution satellite (MOIRE) [2].

\footnotetext{
*Corresponding author: 3327763546@qq.com
}

Stability of mechanical and thermal of payload optical system structure and attitude stablity control are most important key points for GEO optical image satellite.With much larger primary mirror size and focal length,much longer lens hood over LEO, the payload camera presents inevitably a much higher requirement of its structure deformation \& stability accuracy result from mechanical and thermal, and also attitude control accuracy, meanwhile, also longer integration time needs higher attitude stability control precision associated with optical axis pointing,for example, attitude control accuracy of Geo-Oculus should be greater than 29nrad (MTF $\geq 0.95)[3] .1 \mathrm{E}-4^{\circ} / \mathrm{s}$ for over 10 meter resolution and greater than 5.6E-3" (arcsecend) of peak-peak value for high frequency micro-vibraton, it is far greater than LEO optical satellite, and enhanced the development difficulties greatly.Over past years, some of major spacecraft manufacturers or enterprises have been strenthening the technical research and found various ways to minimize micro vibrationa and thermal coupling, shch as Hubble telescope has token the measure of assembly bearing ball more exactly and installation of isolator device for each attitude control actuator to attenuate disturbance influence on picture quality. The attitude accuracy of Chandra $\mathrm{X}$ ray space telescope has been improved obviously by using of isolation system. In order to suppression of distubance induced by actuator, JWST 
space telescope adoptes two stage isolation device, and one is for actuators, another for camera.

Based on MTF analysis and identification of key structure influence factors for image quality guarantee under complex space circumstance and taking a $10 \mathrm{~m}$ resolution GEO remote sensing satellite as a example, this paper conducts some numerical stimulation with influence effect for the identified structure affecting factors, and presents some strategies or suggestions for mitigation micro-vibration and mechanical \& thermal interaction between platform and camera.

\section{Satellite model}

\subsection{Configuration overview}

Typical on-obrital configuration of GEO high spatial resolution optical imaging satellite is illustrated in Fig 1.The spacecraft equipped with large dimension camera, solar-array, picture data transmission antenna etc.The camera with large optical bench usually is mounted above platform, and its optical axis points toward earth. salor-panels are allocated symmetrically along Y-direction (points to South/North earth pole).To avoide obstruction of field of view,picture data transmission antenna and salor-panels have larger size generally.As for $10 \mathrm{~m}$ spatial resolution, the primary mirror diamater is estimated about over $3 \mathrm{~m}$, length of lens hood subjected to duration of sunlight entering field of optical view, may exceed over $10 \mathrm{~m}$ probably.

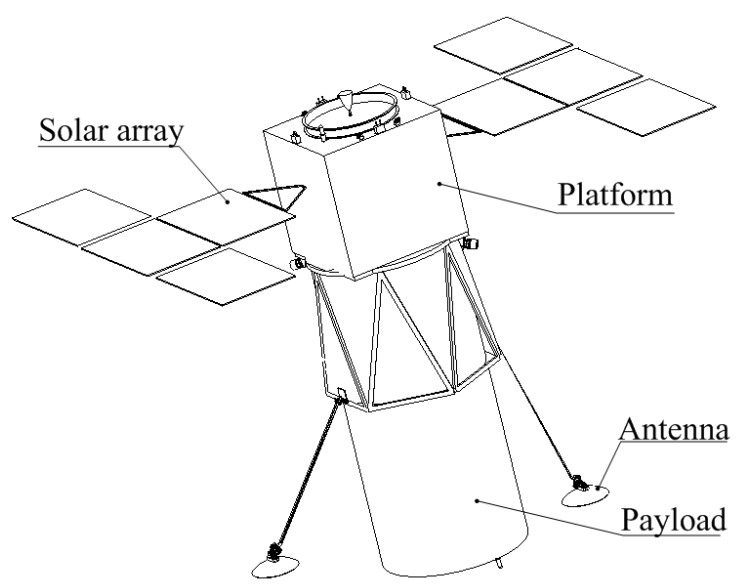

Fig.1 Schematic view of typical Geo imaging satellite cnfiguration

\subsection{Platform-Camera Connection}

Connection structure between platform and camera is a critial point of satellite structure design, and affects directly picture quality through static and dynamic coupling. Because of existing a large difference of structure accuracy between camera and satellite platform with camera much higher accuracy than platform, thus the connection structure plays a important role to accommodate their deformation.

At present,the most common joint ways mainly include following three fashions: multi-bolt fix connection, hinge with lock-release connection, multi-position elastic connection. Multi-bolt fix connection is chiefly used for earlier \& lower resolution optical image satellites, and it has a stronger mechanical and themral interaction or coupling between platform and payload, platform' structure deformation resulted from gravity release and themral will directly impact camera structure, and lead to influence on optical system.the hinge with lock-release connection way is that all of the connetion positions are fixed with pyrotechnicals device except for hinge point during launch process to enhance the bearing ability, after the satellite injected into obrit,then unlocked the fixed bolts to release redundant constraints,only remian few hinges to joint,this mode could reduce effectively or eliminate redundant constraints of rotation freedom degree, but translation constraint exists still in plane. Multiposition elastic connection style may reduce considerably dynamic response during launch course, also migitate influence mutually from mechanical and thermal between plateform-payload,but it is difficult for control the effect usually.

\section{Identification of critical structure factors to affect image quality}

\subsection{Major specfication analysis}

MTF (modulation tranfer function) is the widely used and authoritative criterion with picture quality,so the paper will adopt MTF as image quality judgement criterion and make analysis to major specfication.According to optiacl tranfer chain ,the total MTFis expressed mathematically as following[4]:

MTFT $=$ MTF $1 *$ MTF2 $*$ MTF3 $*$ MTF4 $*$ MTF5 $*$ MTF6 $*$ MTF7 (1)

Where, MTF1 represents MTF result by abberrtion, MTF2 MTF7 are for linear motion, low freqency vibration, high freqency vibration, random motion, focal length devation and other factors respectively.

To accounting for main problem, this article will make a assumption of no assembly error .Based on previous experiance, random motion affecting will not be taken into account.

Refer to reference [4], when aberration is up to $0.05 \lambda$, 
MTF(Nyquist) will decrease to $90 \%$ approximately, and $85 \%$ for $0.07 \lambda$. the reason lead to aberration caused mainly from change of mirror reflection surface shape and relative positions among optical components, so stability of optical bench support structure and maintenance of primary mirror surface shape are essential to satellite performance.

For linear motion, its MTF is expressed as[5]:

$$
\mathrm{MTF}=\operatorname{abs}(\operatorname{sinc}(\pi v f t))
$$

Here, $f$ denotes space frequency, $v$ represents satellite translation speed relative to ground target, $t$ is integration time. As target image shifts to exceed 0.3 pixel on focal plane, MTF will decrease approximately $0.5 \%$, and for 10meter spatial resolution GEO satellite,the integration time is supposed to be $50 \mathrm{~ms}$, then require angular speed is not allowed greater than $10^{-40} / \mathrm{s}$.

When integration time less than a vibration cycle, usually is called as low frequency vibration, its affectting to picture quality is more complex, and not a exact formula to discribe. Becouse of difference of start time and duration of integration,the influence appears some of randomness.If considering worst result, the MTF could be expressed below[6]:

$$
\mathrm{MTF}=\operatorname{sinc}(\pi f a)
$$

For (3), $a$ is blur spot diameter,maximum value located at center of the blur spot, $\mathrm{a}_{\max }=2 \mathrm{Dsin}\left(\pi t_{e} / T\right), f$ is space frequency, $\mathrm{D}$ is amplitude of sine vibration. If $\mathrm{D}$ $=0.005 \mathrm{~mm}, t / T=0.25$, then MTF will approach to $33 \%$ (at $f=60 \mathrm{lp} / \mathrm{mm})[6]$. it shows that low frequency vibration has a large affect on MTF. With increasing of vibration frequency, greater of amplitude and longer of integration time, MTF will decrease more obviously, and more affecting on picture quality.

When integrate time is longer than vibration cycle, usually is called as high frequency vibration. MTF with high frequency vibration can be calculated by following expression (4):

$$
\mathrm{MTF}=\mathrm{J}_{0}(2 \pi f a)
$$

$\mathrm{J}_{0}$ is zeroth Bessel function. When target image shifts exceeds 0.1 pixel on focal plane, the MTF will decrease approximately $5 \%$. As for 10 meter spatial resolution GEO spacecraft, its maximum angular velocity should not greater than $0.0056^{\prime \prime}$.

\subsection{Identification of structure affecting factors}

The above analyses give that the factors lead to MTF decrease include mainly two aspects with static deformation and dynamic vibration.

Mechanical and thermal coupling between platform and payload/camera include gravity, over-constraints' relief and thermal deformation, and have an important role on stability of optical system. Considering the deviation of focal length could be corrected by internal special adjustment mechanism. so the article will focus on analysis of coupling deformation between platform and payload with mechanical and thermal in next sections.

Attitude vibration within control frequency band could be approximated to much smaller linear motion satisfying image performance requirements; Although some flexible components' frequency is lower such as solar-panel, data transmission antenna,but is above control frequency, are main sources of low frequency sine motion. High frequency sources mainly come from rotating mechanism such as CMG, reaction wheel(RW), SADA (solar panel drive unit) etc, its effect involve rigid motion, as well as elastic dynamic, will be discussed detailedly in the following sections.

\section{Stimulation and analysis}

\section{1) Analysis of mechanical and thermal coupling}

\subsection{1 gravity-unloading deformation}

For satellite and camera, the process with manufacture, assembly, accuracy measurement and calibration is always implemented in gravity field. furthermore, the components' material, mass and stiffness distribution are also different in general because of different accuracy need. After the satellite is put into orbit,the gravity will be released and lead to various structure deformation with platform, camera and connecting parts. The mutual coupling constraint may conduct a greater additional shape deviation of primary mirror, change of relative position and angle of components with optical system, caused to lower MTF.

To assessing effect of mechanical and thermal coupling, the FEA(Finite Element Analysis) stimulation and analysis with $10 \mathrm{~m}$ resolution GEO optical image satellite are performed both for eight-points fix connection mode and three-points hinge connection mode (locked on launch, unlocked after separation of satellite and rocket). Platform structure material is mainly consist of sandwich ect, core is $\mathrm{Al}$ honeycomb,thickness contains $25 \mathrm{~mm}$ and $30 \mathrm{~mm}$, facesheet include C-fiber or Aluminium alloy, thickness is set to $0.3 \mathrm{~mm}$, support is set as titanium alloy, payload weighted about $1600 \mathrm{~kg}$, total weight is proximately $7000 \mathrm{~kg}$ with satellite. FEA model is shown in Fig.2.Stimulation results are figured in Fig.3 and Fig.4.

The analysis could be summarized as following:

1) For 8-points fixed connection mode,deformation of 
interface mainly include lateral tension, bending deformation and rigid rotation.Deformation of bending is obviously greater than lateral tension, and appear lower in middle part and higher outside.

2) For 3-points hinge connection mode, deformation of interface mainly include lateral tension and rigid rotation, the bending deformation is lower relatively to rigid rotation angle with about $11^{\prime \prime}$.

3 ) Bending and rigid rotate are the main affect factors obviously, three-points hinge connection is far better than eight-points fix connection.As for tension deformation in plane, it can be controlled or improved through stiffness match design between platform and payload.Rigid rotate will result in optical-axis pointing error,could be corrected or improved by ground calibration.
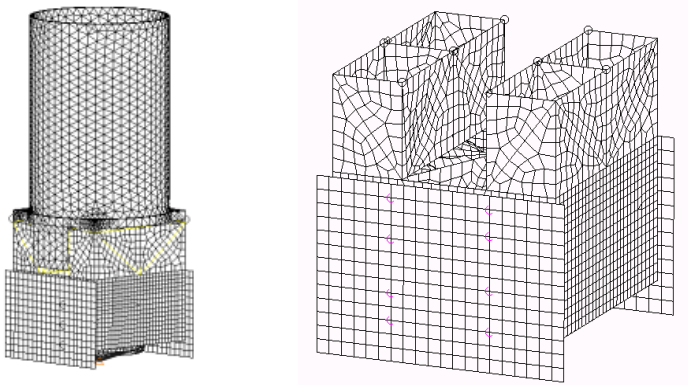

Fig. 2 FEA model

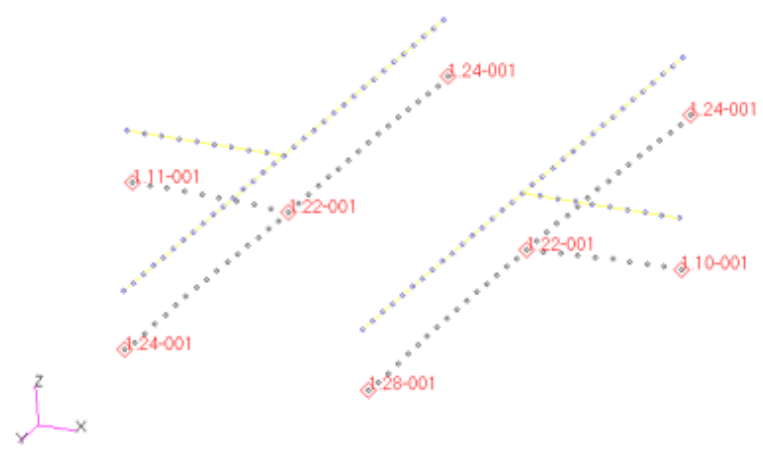

Fig. 3 Deformation for 8-points

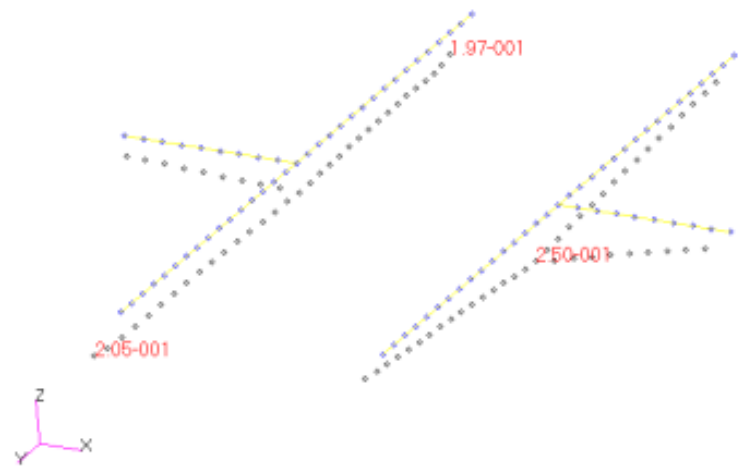

Fig. 4 Deformation for 3-points

In practical application, in term of analysis result, the viable mitigation methods of gravity unloading lead to deformation mutually is suggested that options could include such as single-hinge joint mode (multi-points lock and unlock on orbit) 、 multi-points flexible connection and release redundant degree of freedom,etc.the above strategy was proven to be effective by some of in-orbit flight.

\subsubsection{Thermal deformation analysis}

In-orbit flight, there exists a greater difference of temperature control accuracy between platform and payload, the temperature control range of platform is far less than payolad,temperature within platform always occurs larger fluctuation as change of orbital position and different operation mode.The wave of heat flow density will produce connection structure thermal deformation and stress coupling,eventually leads to size variation of support structure of optical system.

The following performs a thermal deformation simulation and analysis for a typical case for 3-points hinge joint mode, the ground assembly temperature is assumed as $20^{\circ}$, during orbital operation,platform panels' temperature distribution is set to to be in range of $15^{\circ}$ $\sim 34^{\circ}$, camera temperature is controlled within $20^{\circ} \pm 1^{\circ}$.

Thermal FEA model is shown in Fig.5, stimulation results for deformation and displacement at joint positions are offered in Fig.6 and table 1 individually. The analysis exhibits that the thermal changes mainly contribute to interface lateral deformation,maximum change value of relative position is close to $1 \mathrm{~mm}$, have a greater effect on optical structure.

To reducing thermal coupling deformation, use a number of elastic panel with little height instead of two hinge joint devices,the analysis result is illustrated in Fig. 6 and table 1 respectively, the strain energy of elastic device and comparison are listed in table 2 before and after deformation release device installation. The results obviously show the better improvement by use of elastic device.

Based on above analysis, elastic connection scheme is proposed that the interaction deformation could be greatly improved. The method was already validated in some experiments. 


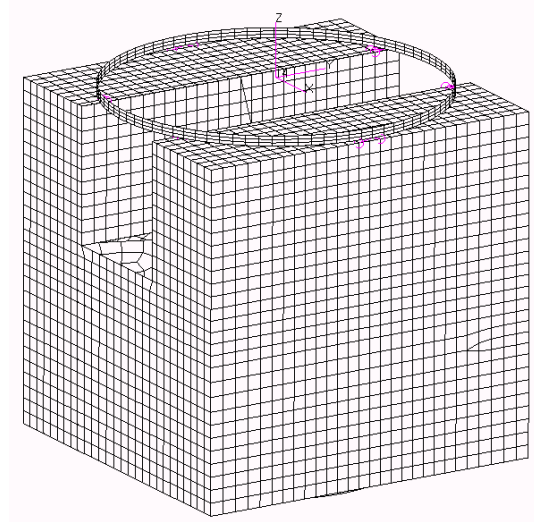

Fig. 5 Thermal FEA model

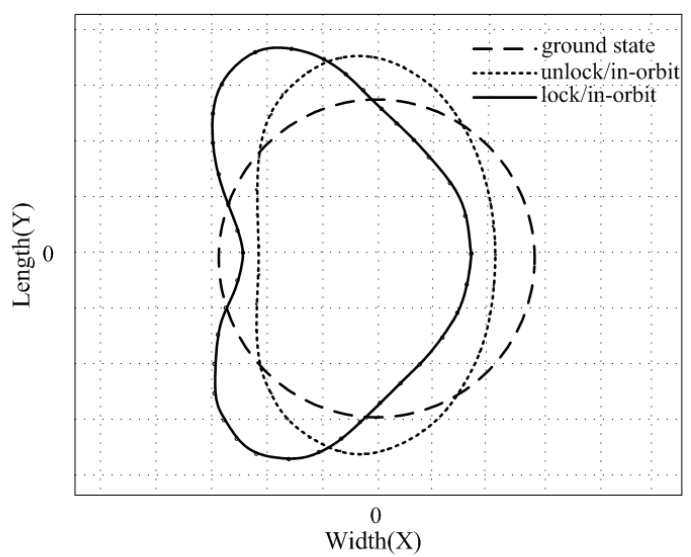

Fig. 6 Deformation situation

Table1 Stimulation results

\begin{tabular}{|c|c|c|c|}
\hline \multirow[b]{2}{*}{$\begin{array}{l}\text { Joint } \\
\text { location }\end{array}$} & \multicolumn{2}{|c|}{ Displacement at joint point $/ \mu \mathrm{m}$} & \multirow[b]{2}{*}{ Ratio } \\
\hline & $\begin{array}{l}\text { Before mount } \\
\text { relief }(\mu \mathrm{m})\end{array}$ & $\begin{array}{l}\text { After mount } \\
\text { relief }(\mu \mathrm{m})\end{array}$ & \\
\hline 1 & 643 & 42 & $6.5 \%$ \\
\hline 2 & 200 & 56 & $28 \%$ \\
\hline 3 & 176 & 51 & $29 \%$ \\
\hline
\end{tabular}

Table2 strain energy

\begin{tabular}{|c|c|c|}
\hline $\begin{array}{c}\text { After } \\
\text { deformation } \\
\text { relief }(\mathrm{mJ})\end{array}$ & $\begin{array}{c}\text { Before deformation } \\
\text { relief }(\mathrm{mJ})\end{array}$ & Ratio \\
\hline 7 & 56 & $13 \%$ \\
\hline
\end{tabular}

\subsection{Affect analysis of low frequency vibration}

As stated above,the components with lower natural frequencies include mainly solar-panel, lens hood, data transmission antenna,etc.Take GEO 10m-spatial resolution optical satellite as a example, if integrate time is set as $50 \mathrm{~ms}$, attitude angle amplitude is $1 \times 10^{-40}$, and $0.25 \mathrm{~Hz}$ with first natural frequency of solar-panel, then, MTF will decrease up to $83 \%$. As solar-panel frequency increasing to $0.6 \mathrm{~Hz}, \mathrm{MTF}$ will be $30 \%$. It indicates that MTF will decrease distinctly as frequency and attitude angle amplitude increase.

The following Fig.7 present test disturbance momentum Mx ( about $\mathrm{X}$ axis) and My (about $\mathrm{Y}$ axis) time-domain curves of a solar-panel at SADA mounting position while SADA operating. The curves involve a $0.25 \mathrm{~Hz}$ and $0.65 \mathrm{~Hz}$ frequency correspondence to $\mathrm{X}$ axis and $\mathrm{Y}$ axis respectively. Calculation results of maximum attitude angle amplitude associated with MTF for roll and pitch axis are listed in table 3 , it is seen that MTF drops about $1 \%$ and $15 \%$ respectively.

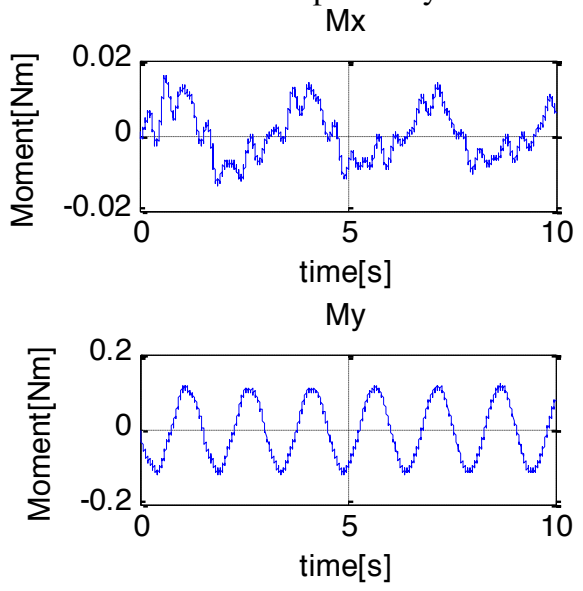

Fig.7 SADA disturbance momentum at mounting position

Table3 Max angular disp.(deg)within integration time

\begin{tabular}{|l|l|l|}
\hline Integration time $(\mathrm{ms})$ & $\mathrm{X}$ & $\mathrm{Y}$ \\
\hline 50 & $2.3 \mathrm{e}-005$ & $3.90 \mathrm{e}-005$ \\
\hline
\end{tabular}

By the theoretical formula, experience and related experiments, the strategy with reducing low frequency disturbance is suggested including measures of decreasing flexible components' natural frequency, lowing vibration amplitude or shortening integration time. Enlarging satellite interia or shortening the distance between flexible components such as solar-panel,data transmission antenna and centroid of satellite are effective methods which could degrade disturbance amplitude. Integration time is relevant greatly to payload/detector design and manufacture ability, should take some optimization methods to improve its performance.

\subsection{Affect analysis of high frequency vibration}

\subsection{1 characteristic of disturbance sources}

Micro-vibration sources mainly contain some rotating mechanisms such as CMG and RW etc.Fig.8 provide a 
set of typical test disturbance time-domain curves of RW and $\mathrm{CMG}$ at mountingposition.RW and CMG involves single frequency $60 \mathrm{~Hz}$ and $100 \mathrm{~Hz}$ individually,moreover,there exist a number of times-frequencies also.
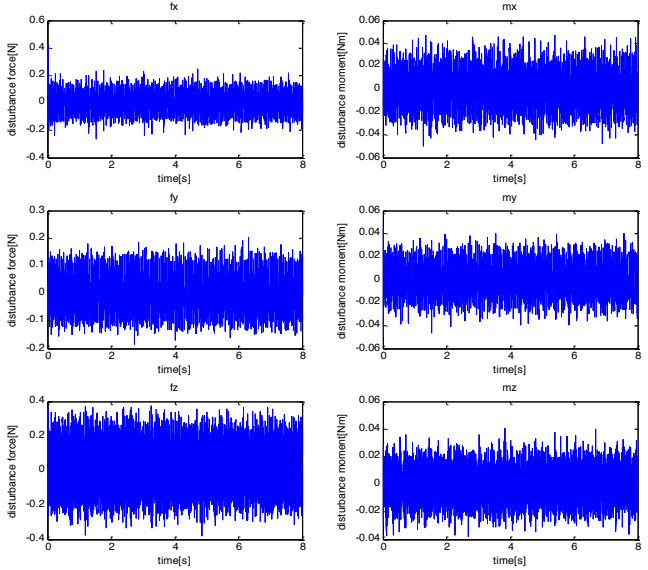

RW time-domain curves
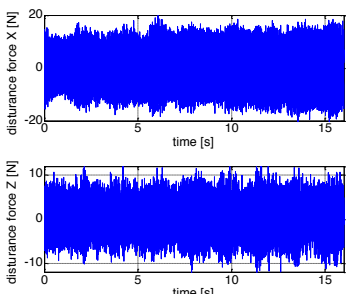

吝
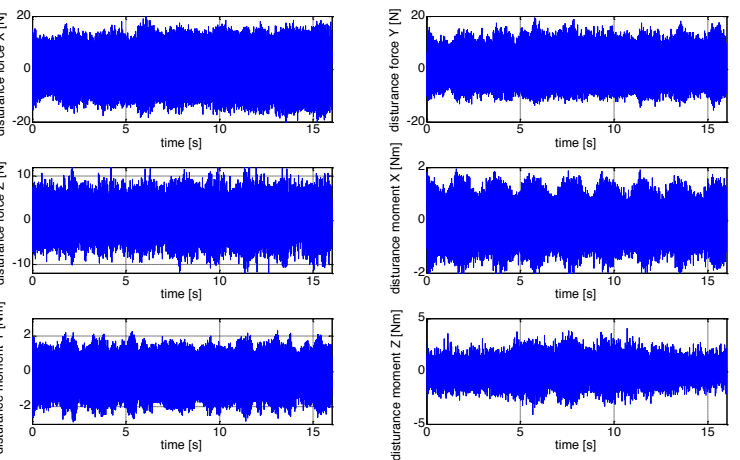

CMG time-domain curves at $0^{\circ}$ gimbal angle

Fig.8 Typical disturbance time-domain curves

\subsubsection{Affect analysis of high frequency vibration}

Providing the above GEO-10m example (4.1 section) equipped with three RW and three CMG, their angular momentum points to three satellite coordinate axis individually, all of RWs and CMGs placed on satellite bottom panel.FEA model is shown as Fig.9, PATRAN/NASTRAN is employed to carry out the stimulation and analysis with dynamic response.

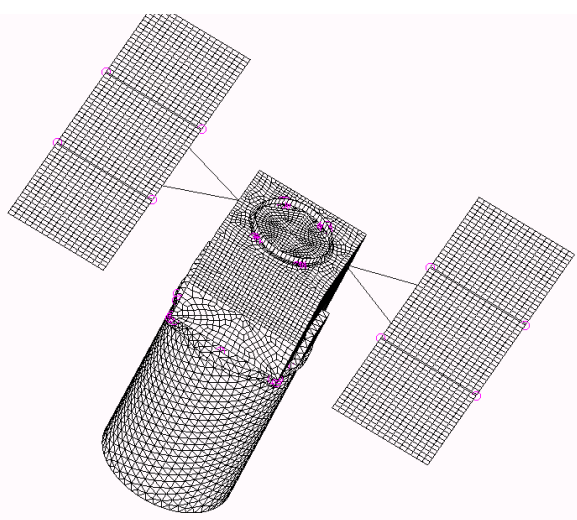

Fig.9 FEA model in-flight

Fig.10 present angle displacement response curves of $\mathrm{X}$ axis ( roll ) , $\mathrm{Y}$ axis ( pitch) , $\mathrm{Z}$ axis ( yaw ) in frequency-domain respectively.The results reveal that:

1) Micro-vibration with RW will produce optical axis a 8.46E-02" (Max value) angle displacement,and far greater than the requirement with $5.6 \mathrm{E}-3^{\prime \prime}$, the Max value occurred at $60 \mathrm{~Hz}, \mathrm{MTF}($ Nyquist) decrease over $40 \%$, have a distinct effect on picture quality.

2) Disturbance effect caused by CMG is more greater than RW, it will be greatly possible to result in useless of collected pictures if some measures are not taken.

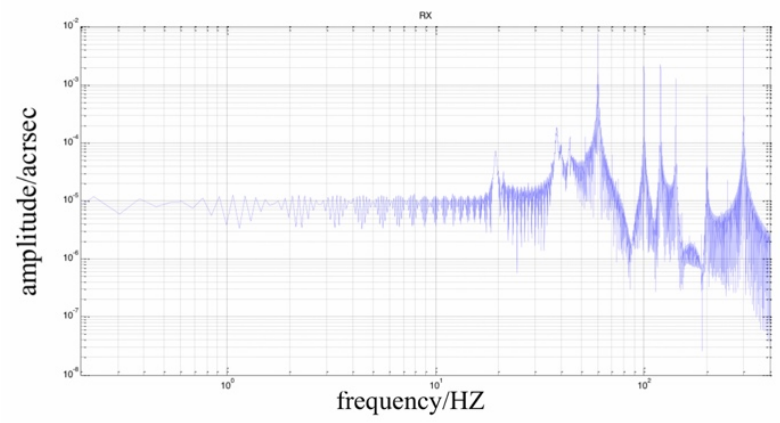

$\mathrm{RX}$

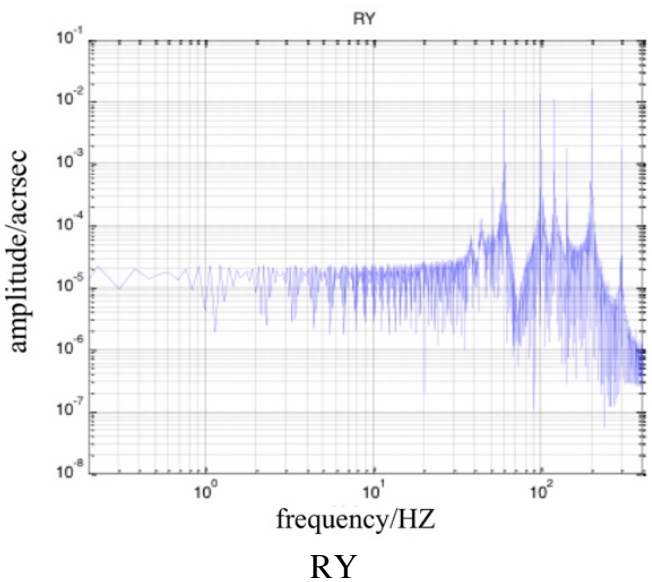




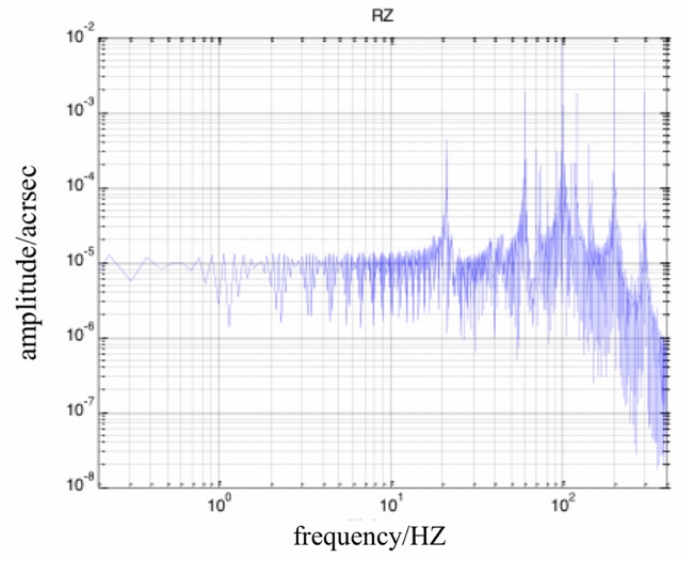

RZ

Fig.10 Dynamic response curve of optical axis in frequency-domain

\subsubsection{Suppression scheme}

The following schemes to reducing micro-vibration amplitude suggested:

1) Configure Extra-low-noise actuator

Comparing with the common mechanical RW, magnetically suspend momentum wheel (MSMW) has an advantage of much less disturbance noise, it has applied already on some satellites such as SPOT-5 series etc. China has been also making related research and developing with MSMW. The Fig.11 lists a test disturbance results of a sample about $\mathrm{X}$ axis and Yaxis in course of speed decreasing from 5200rpm to 0rpm,Max disturbed force happened at 5200rpm and less than $4 \mathrm{~N}$. Calculation shows that Max rotation angle of optical axis could drop up to 5.1E-3" (listed in table 4)after mechanical RW is insteaded by MSMW, here assumed optical structure rigid.it has been validated that it is a effective solution route by use of MSMW.

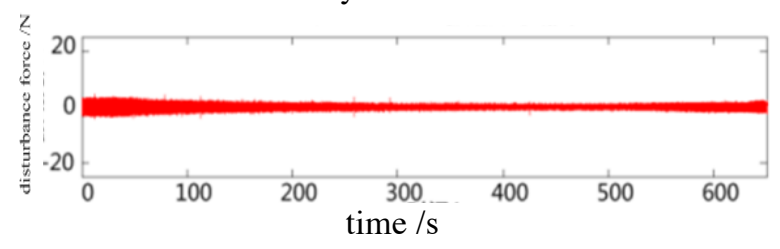

Fx disturbance force

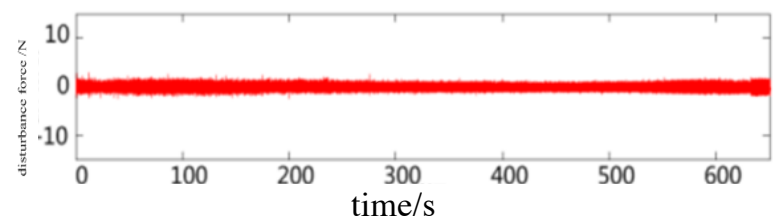

Fy disturbance force

Fig.11. Disturbance load during speed decreasing from 5200rpm to 0rpm
Table 4 Max angular displacement (deg) within integration time $(\operatorname{arcsec})$

\begin{tabular}{|l|l|l|}
\hline integration time $(\mathrm{ms})$ & $\mathrm{RX}(\operatorname{arcsec})$ & $\mathrm{RY}(\operatorname{arcsec})$ \\
\hline 50 & $2.40 \mathrm{E}-3$ & $5.1 \mathrm{E}-3$ \\
\hline
\end{tabular}

to actuator

Isolation is a popular and effective way to reduce disturbance noise, the key consideration with this method focus on frequency match design which isolation frequency must be as far apart from the actuator rotate excited frequency as possible.Now,to address the effect of mothed, a isolation model is constructed as example, individual stiffness in three direction is set to identical as $2.0 \mathrm{e} 5 \mathrm{Nm}^{-1}$,damping coefficient is $50 \mathrm{Nsm}^{-1}$, the excited load comes from $25 \mathrm{NmsCMG}$ test data.Table 5 offers the transfer force at joint location between isolation and platform for before and after isolated,Fig.13 present rotation angle time-domain response curves of optical axis.it can be seen that the Min decay ratio reach up to $80 \%$, Max rotation angle of optical axis decreased to 4e-003" approximately after isolation use,vibration amplitude could get a obviously improvement.

Table5 Transfer force comparison between before and after isolated

\begin{tabular}{|l|l|l|l|}
\hline force & $\begin{array}{l}\text { befor isolation } \\
(\text { RMS })\end{array}$ & $\begin{array}{l}\text { after isolation } \\
(\text { RMS })\end{array}$ & $\begin{array}{l}\text { decay } \\
\text { ratio }\end{array}$ \\
\hline Fx $(\mathrm{N})$ & 8.1 & 0.9 & $89 \%$ \\
\hline Fy $(\mathrm{N})$ & 4.2 & 0.5 & $88 \%$ \\
\hline Fz $(\mathrm{N})$ & 2.10 & 0.2 & $91 \%$ \\
\hline $\mathrm{Mx}(\mathrm{Nm})$ & 0.4 & 0.03 & $92 \%$ \\
\hline $\mathrm{My}(\mathrm{Nm})$ & 0.7 & 0.08 & $89 \%$ \\
\hline $\mathrm{Mz}(\mathrm{Nm})$ & 0.5 & 0.1 & $80 \%$ \\
\hline
\end{tabular}

3) Isolate between platform and payload

Similary, connecting with elastic device could block or suppress actuator perturb load.Taking elastic stiffness as $8 \mathrm{e} 5 \mathrm{Nm}^{-1}$ and viso-damping coefficient $50 \mathrm{Nsm}^{-1}$ along three setallite axis individually for the isolator device respectively,Table6 gives Max angle displacement comparison before and after isolated at camera mountingposition.it shows the min decay ratio is over $50 \%$. This measure may be one of options also.

Table 6 Max angular displacement (deg) within integration time $(\operatorname{arcsec})$

\begin{tabular}{|l|l|l|}
\hline & $\mathrm{RX}(\operatorname{arcsec})$ & $\mathrm{RY}(\operatorname{arcsec})$ \\
\hline before isolation & $7.1 \mathrm{e}-003$ & $5.7 \mathrm{e}-003$ \\
\hline after isolation & $2 . \mathrm{e}-003$ & $2.8 \mathrm{e}-003$ \\
\hline decay ratio & $72 \%$ & $51 \%$ \\
\hline
\end{tabular}




\section{Summary}

Based on main parameters analysis related structure static and dynamic stability performance with GEO optical imaging quality guarantee, the paper performs a identification and stimulation of key structure factors and its effect,and present some suggestions to reduce disturbance noise.The analysis results can be concluded as:

1) Connection structure characteristic of platform and payload is very important to imaging performance.The stronger mechanical and thermal coulpling mutually between platform and payload will directly affect picture quality, should take a consideration seriously in structure design.

2) To reduce effect of mechanical and thermal deformation coupling,some methods could be applied such as hinge joint mode (multi-points lock and unlock on orbit)、 multi-points flexible connection and release redundant degree of freedom,etc..

$3 \mathrm{RW}$ and CMG are some of major micro-vibration sources, have a much greater influence on MTF,specially for GEO optical imaging spacecraft, if not controlled,it maybe result in system mission failure.So it is a critial point of structure design or sysem design also.

4) Main measures to reducing the disturbance is suggested including decreasing excited load amplitude such as use low-noise MSMW 、 block disturbance tansfer path such as isolation for acuators or payload etc.If necessary,some of these measures could be combined to use.

\section{References}

1. 1.M.Lingjie,G.Ding,T.Menghui,W.Qi,Spacecraft Recovery \&Remote Sensing,Vol.37,No4,1-5(2106)

2. .G.Linghua,D.Zheng,T.Jiasheng,etal..Spacecraft Recovery \&Remote Sensing,Vol.31,No6, 23-30.(2010)

3. T.Xiao-ping,L.Xiao,X.Dong-Lin.Optics and Precision Engineering,Vol.21.No.8 ,2169 2179(2013)

4. H.Chang-yuan,Optics and Precision Engineering, Vol.23,No 1,2-9(2015)

5. Y.Xun,S.Wuhan,W.Ying,M.Lin.Journal of Applied Optics, Vol.01,NO6, 928-932(2013)

6. L.Haoyang,L.Zhaojun,X.Pengmei.Computer Simulation. Vol.29, No.1, 25-27(2012) 